\title{
Using dot blot with immunochemical detection to evaluate global changes in SUMO-2/3 conjugation
}

\author{
Markéta Častorálová, Tomáš Ruml, Zdeněk Knejzlík \\ Department of Biochemistry and Microbiology, Institute of Chemical Technology, Prague, Czech Republic
}

Small ubiquitin-related modifier-2/3 (SUMO-2/3) is a member of the ubiquitin-like (Ubl) protein family. Conjugation of SUMO-2/3 to target proteins is influenced by various stress conditions and chemical inhibitors. SUMO-2/3 conjugation may serve as a neuroprotective mechanism and may play a role in protein quality control. A method for screening global changes in SUMO-2/3 conjugation would facilitate further research of SUMO-2/3 cellular function. Here we show that dot blot with immunochemical detection allows evaluation of changes in global cellular SUMO-2/3 conjugation and offers an alternative to more laborious Western blot analysis. The method is based on a change of SUMO-2/3 signal intensity upon its conjugation. The dot blot analysis presented here is a timesaving method that enables screening of large numbers of samples and easy statistical evaluation of the results.

BioTechniques 52:1-4 (September 2012) doi 10.2144/000113925

Keywords:_SUMO-2/3; SUMO-2/3 conjugation; dot blot

Small ubiquitin-related modifiers (SUMOs) belong to the ubiquitin-like (Ubl) protein family; they are structurally similar to ubiquitin and have the ability to conjugate with target proteins. Vertebrates encode three SUMO isoforms: SUMO-1, SUMO-2, and SUMO-3. Mature SUMO-2 exhibits approximately $97 \%$ sequence identity to mature SUMO-3 (together designated further as SUMO-2/3). All three SUMO isoforms are conjugated to target proteins by common enzymatic components of the SUMO conjugation pathway $(1,2)$; the reversible nature of SUMO attachment provided by SUMO proteases (reviewed in 3) makes SUMOs flexible posttranslational modifiers. SUMOylation, conjugation of SUMO to target proteins, plays role in many cellular processes such as subcellular localization, transcription regulation, DNA replication, and repair $(2,4)$.

Conjugation of SUMO-2/3 to cellular targets increases under various stress stimuli $(5,6)$ or under proteasomal inhibition $(7)$. Even though the proteome of SUMO-2/3 targets was identified in heat-shocked cells (8) and in cells with inhibited proteasomal activity $(9,10)$, the precise role of stress-induced SUMOylation still is not well understood. However, an association between ubiquitin-targeted degradation and a protective role under stress conditions has been proposed (7-9). In the brain, SUMO-2/3 conjugation was shown to be strongly activated in hibernating animals during the torpor phase $(11,12)$, and it was suggested that this served as a protective response by shielding neurons from damage induced by low blood flow and substrate deprivation (12). Further, SUMO-2/3 conjugation is activated during deep hypothermia (13) and a significant increase in the level of SUMO2/3-conjugated proteins was found in the brains of animals subjected to transient global or focal cerebral ischemia (14). Since SUMO-2/3 knockdown was shown to increase vulnerability of neuronal cells to oxygen and glucose deprivation (OGD), the activation of SUMO-2/3 conjugation was suggested to serve as an endogenous neuroprotective stress response (15). In this context, it was reported that enhanced
SUMOylation during OGD in cultured hippocampal neurons is not attributable to increased SUMO gene expression (16). In addition to these findings, it was proposed that SUMO-2/3 conjugation was connected to protein quality control at a cellular level in various physiological and pathological processes $(10,17)$. In the near future, we expect to see more studies focusing on the detail of SUMOylation in tissues under different pathological conditions and, eventually, treatment of disease by activation or inhibition of SUMOylation as proposed (15).

The most frequently used technique for detecting changes in SUMO-2/3 conjugation is Western blot analysis, consisting of fractionation of cell lysates by SDS-PAGE followed by electroblotting of separated proteins from the polyacrylamide gel to a nitrocellulose membrane and detection of SUMO-2/3 with a specific antibody. The qualitative marker of covalent SUMO-2/3 conjugation to protein targets is a decrease of SUMO-2/3 electrophoretic mobility, often corresponding to a molecular weight ranging from 60 to $200 \mathrm{kDa}$. However,

\section{Method summary:}

We show that dot blot analysis is an efficient and valuable method for quantifying changes in SUMO-2/3 conjugation in cell cultures and animal tissues. The method readily distinguishes differences in total protein concentration between individual samples, requires less time than Western blot analysis, enables researchers to screen large numbers of samples, and allows for easy evaluation of the results. 
since large sets of proteins may be modified by SUMO-2/3 $(18,19)$, a smear is often observed in Western blot analysis $(5,10$; Figure 1). The signal intensity of the smear corresponding to conjugated SUMO-2/3 is dramatically increased compared with the original signal from unconjugated SUMO-2/3. Although the higher signal intensity of conjugated SUMO-2/3 has been observed many times $(5,10)$, the nature of this phenomenon has not been discussed. One possible explanation is that immunoreactive epitopes in SUMO-2/3 become easier for antibodies to recognize following conjugation because of immobilization of SUMO-2/3 with its protein targets on the membrane. Other possibilities are that SUMO-2/3 conjugates transfer more readily from the polyacrylamide gel than free SUMO-2/3 or that free SUMO-2/3 has extremely low-binding affinity for the membrane. Here we used heat shock and treatment with proteasomal inhibitor MG132 as model conditions to induce accumulation of SUMO-2/3 conjugates in HEK 293T cells and analyzed SUMOylation by Western blot analysis on nitrocellulose $(0.45 \mu \mathrm{m})$ or PVDF $(0.2$ $\mu \mathrm{m})$ membranes (Figure 1, lanes 2 and 3); PVDF membranes proved to be more suitable for simultaneous detection of free SUMO-2/3 and its conjugates under the same exposure time. Parallel analysis of lysates where SUMO-2/3 was deconjugated by the SUMO-specific isopeptidase Ulp1 showed that the increased signal intensity of SUMO-2/3 is caused exclusively by SUMO- $2 / 3$ conjugation and not by increased expression because similar signal levels from deconjugated SUMO-2/3 were detected under all tested conditions (Figure 1, compare lanes 4-6). Surprisingly, similar qualitative findings for SUMO-2/3 conjugate intensities were observed when lysates were directly dot-blotted onto nitrocellulose or PVDF membranes and probed with antibodies against SUMO-2/3 (Figure 1). The signal change caused by conjugation did not depend on membrane or antibody type because $\mathrm{N}$-teminally $\mathrm{HA}$ fused SUMO-3 protein expressed in HEK 293T cells showed identical behavior using mouse monoclonal anti-HA antibody (data not shown). The samples used for Western blot analysis contained intentionally high total protein concentrations to obtain a visible decrease of free SUMO-2/3 levels as an indicator of increased conjugation. Although unconjugated SUMO-2/3 was detectable by Western blot, almost no signal was observed by dot blot analysis either on nitrocellulose or PVDF membranes (Figure 1, lanes 4-6). This observation was surprising considering
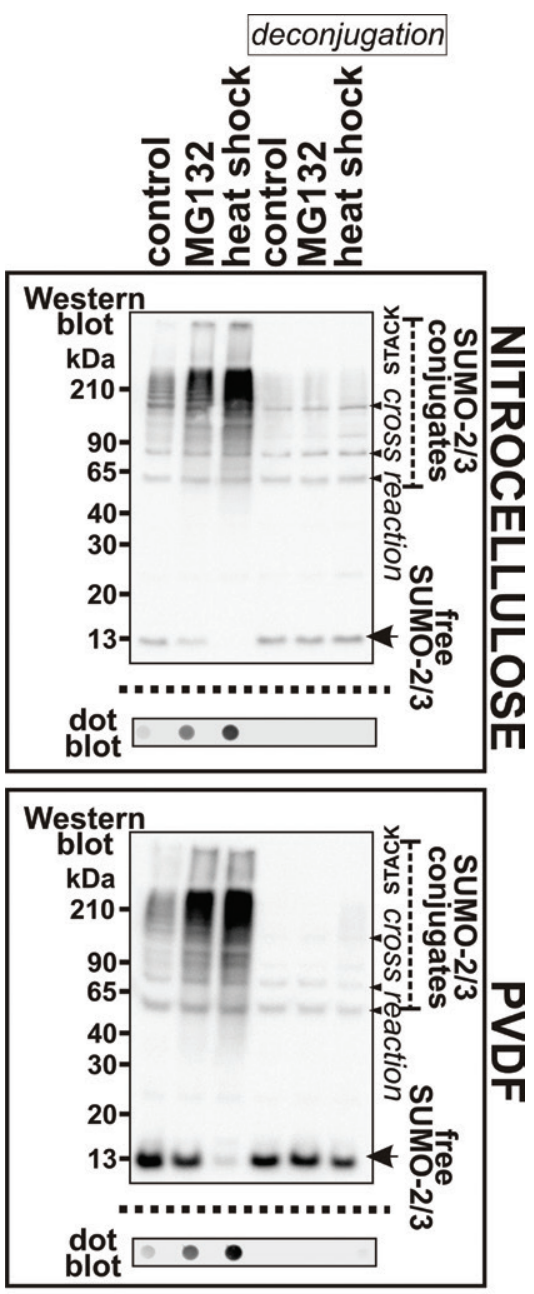

GAPDH ------

lane: 123456
Figure 1. Immunodetection of SUMO-2/3 conjugates on nitrocellulose and PVDF membranes. HEK 293T cells were cultivated in Dulbecco's modified Eagle's medium (DMEM, Sigma-Aldrich, St. Louis, MO, USA) supplemented with $10 \%$ fetal bovine serum and vitamins (both from Invitrogen, Life Technologies, Grand Island, NY, USA) on a $35 \mathrm{~mm}$ Petri dish to $80 \%$ confluence. Heat shock was performed by cultivation of cells at $42^{\circ} \mathrm{C}$ for $1 \mathrm{~h}$ (lines 3 and 6 ). Proteasomes were inhibited using $10 \mu \mathrm{M}$ MG132 (PeptaNova, Sandhausen, Germany) for $2 \mathrm{~h}$ at $37^{\circ} \mathrm{C}$ (line 2 and 5). Both treated and control cells were released from the cultivation surface by $1 \mathrm{~mL}$ of cultivation media and two $400 \mu \mathrm{l}$ cell suspension aliquots were collected. Cells were pelleted at $1,000 \times \mathrm{g}$ for $1 \mathrm{~min}$, medium was discarded, and cell pellets were lysed as follows: to samples shown in Lanes 1, 2, and 3, $100 \mu \mathrm{l}$ of reducing SDS-PAGE Laemli sample buffer (100 mM Tris, pH 6.8, 8\% SDS 24\% glycerol, 0.02\% Coomassie blue G-250, 200 $\mathrm{mM}$ dithiothreitol) were added, lysate was sonicated and boiled for $10 \mathrm{~min}$; in lanes 4,5 and 6 (deconjugation of SUMO-2/3), the cell pellet was resuspended in $50 \mu$ of native lysis buffer $(50 \mathrm{mM}$ Tris- $\mathrm{HCl}, 150 \mathrm{mM}$ $\mathrm{NaCl}$, 0,4\% NP40, 1 mM DTT, 1 mM EDTA, $20 \mu \mathrm{M}$ MG132, $20 \mu \mathrm{g} / \mathrm{mL}$ Leupeptin, 1mM PMSF, pH 8) containing $1 \cup$ of $S$. cerevisiae protease Ulp1 (Invitrogen). The SUMO-2/3 deconjugation reaction took place for $2 \mathrm{~h}$ at room temperature and then $50 \mu \mathrm{l}$ of reducing SDS-PAGE Laemli sample buffer was added; then the lysate was sonicated and boiled for $10 \mathrm{~min}$. For Western blot, samples ( $10 \mu \mathrm{l})$ were analyzed by SDS-PAGE and transferred to nitrocellulose or PVDF membranes in transfer buffer for $1 \mathrm{~h}$ at $100 \mathrm{~V}$. For dot blot, samples $(10 \mu \mathrm{l})$ were diluted in TBS $(20 \mathrm{mM}$ Tris. $\mathrm{HCl}, 150 \mathrm{mM}$ $\mathrm{NaCl}, \mathrm{pH} 8.0$ ) to a final volume of $200 \mu \mathrm{l}$ and spotted onto a nitrocellulose or PVDF membrane using a BioDot SF Microfiltration Apparatus (Bio-Rad, Hercules, CA, USA) according to the manufacturer's instructions. After Western and dot blotting, the membranes were incubated in blocking solution (5\% fat-free milk, $0.1 \%$ Tween-20 in PBS) for $1 \mathrm{~h}$. Endogenous SUMO-2/3 was detected using polyclonal anti-SUMO-2 antibody (Invitrogen) at a 1:750 dilution. Protein level normalization was carried out by rabbit polyclonal anti-GAPDH antibody (Sigma-Aldrich), used 1:10,000 dilution. Secondary anti-rabbit IgG specific HRP-conjugated antibody (Santa Cruz Biotechnology, Santa Cruz, CA, USA) was used at 1:5000 dilution. SUMOylated proteins were visualized by SuperSignal West Femto chemiluminescent substrate (Pierce, Thermo Scientific, Rockford, IL, USA) according to the manufacturer's instructions.

that in addition to free SUMO-2/3, there were three additional cross reactive bands detected with Western blot (Figure 1, lanes 4-6; 20). Independent of the type of membrane, these results indicate that the SUMO-2/3 signal on the dot blot is a function of the level of SUMO-2/3 conjugates. Free SUMO-2/3 is far less detectable by dot blot than by Western blot, possibly because of lower affinity to the membrane, competition with other cellular proteins for membrane binding sites, or shielding of epitopes by other cellular proteins or the membrane. Dot blot previously has been used to detect global cellular levels of SUMO-1 (21). However, because of the increase of SUMO-2/3 signal intensity upon its conjugation, it is not possible to use this method to assess global cellular levels of SUMO-2/3 as it was used for SUMO-1 (21).

Next, we examined the dependence of the Western blot and dot blot signal intensity on the percentage of conjugated SUMO-2/3 (Figure 2). To obtain defined levels of conjugated SUMO-2/3 in total cell lysates, the lysate from heatshocked cells was mixed at various ratios with cell lysate of the same origin but treated with Ulp1 for global SUMO-2/3 deconjugation. The signal intensity of free SUMO-2/3 and SUMO-2/3 conjugates in the individual samples with comparable total protein content was verified decrease of free SUMO-2/3 signal correlated with the theoretical increase of the level of SUMO-2/3 conjugates. However, by Western blot analysis (Figure 2A). The 
A
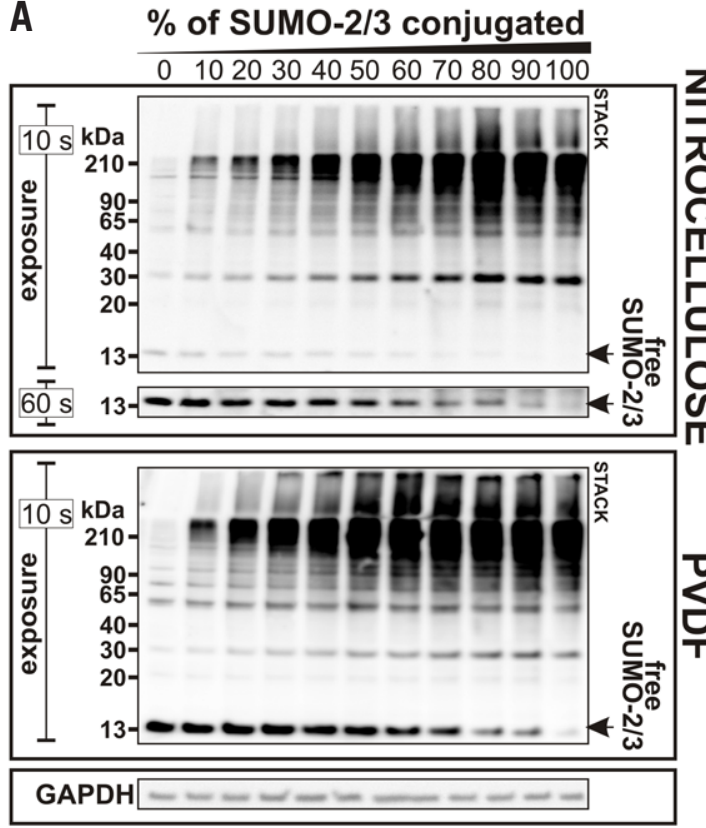

B

\begin{tabular}{|ccccccccccc|}
\hline \multicolumn{8}{|c|}{$\%$ of SUMO-2/3 conjugated } \\
\hline 0 & 10 & 20 & 30 & 40 & 50 & 60 & 70 & 80 & 90 & 100 \\
\hline & 0 & $\bullet$ & $\bullet$ & $\bullet$ & $\bullet$ & $\bullet$ & $\bullet$ & $\bullet$ & $\bullet$ & NITROCELLULOSE \\
\hline & 0 & 0 & $\bullet$ & $\bullet$ & $\bullet$ & $\bullet$ & $\bullet$ & $\bullet$ & $\bullet$ & PVDF \\
\hline
\end{tabular}

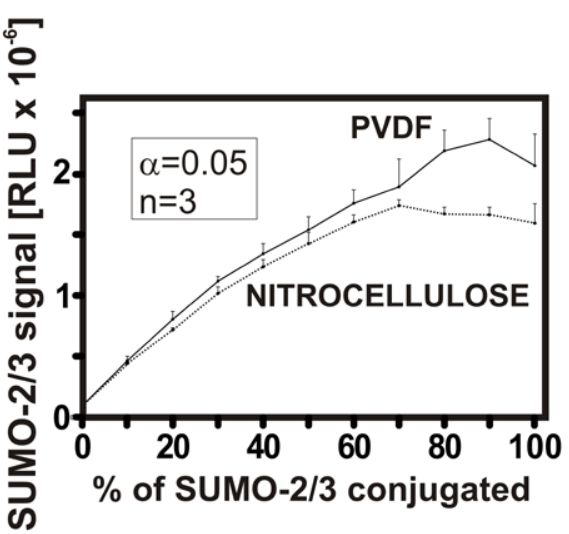

Figure 2. Western blot and dot blot analysis of SUM0-2/3 conjugates. HEK $293 \mathrm{~T}$ cells ( $10 \mathrm{~cm}$ Petri dish) were cultivated at $42^{\circ} \mathrm{C}$ for $1 \mathrm{~h}$. Cells were released by $2 \mathrm{~mL}$ of cultivation medium, divided into two equal parts, and centrifuged (1,000× g, $1 \mathrm{~min})$. For SUMO-2/3 conjugate detection, the cell pellet was lysed in $300 \mu \mathrm{l}$ of Laemli sample buffer. A second pellet was resuspended in $150 \mu \mathrm{l}$ of native lysis buffer for SUMO-2/3 deconujugation (as described in the Figure 1 legend), incubated for $2 \mathrm{~h}$ at room temperature, and then $150 \mu \mathrm{l}$ of Laemli sample buffer was added. The lysates were sonicated and boiled for 10 min. The total cell lysates with conjugated (100\% conjugation) and deconjugated ( $0 \%$ conjugation) SUMO- $2 / 3$ were mixed to generate a series of samples including amounts of conjugated SUMO-2/3 from 0 to 100\%. Samples were analyzed by Western blot (A) or dot blot (B) either on nitrocellulose or PVDF membrane with immunochemical detection. The quantification of dot blots was carried out using TotalLab TL100 software (Nonlinear Dynamics USA, Durham, NC, USA).

for the increase of SUMO-2/3 conjugate, the signal was visible in the interval of $0 \%$ (only unconjugated SUMO-2/3) to approximately $60 \%$ (SUMO-2/3 conjugated on nitrocellulose), and up to $90 \%$ on PVDF (Figure 2). Dot blot analysis showed an approximately linear correleation between the signal and the level of conjugated SUMO- $2 / 3$ in the interval from $0 \%$ to $60 \%$ of SUMO-2/3 conjugated on nitrocellulose and 0 to $90 \%$ conjugated on PVDF, which was in agreement with results obtained by Western blot (Figure 2). Even with reduced protein quantities near the detection limit of dot blot, we observed a constant signal above $60 \%-70 \%$ of SUMO- $2 / 3$ conjugated on nitrocellulose membrane. Based on these results, we recommend using PVDF membranes for evaluation of global changes in SUMO-2/3 conjugation by dot blot.

Finally, we used dot blot to screen for changes in global SUMO-2/3 conjugation in HEK 293T cells grown under different conditions (Figure 3). Each sample was tested in four replicates using dot blot and the signal intensities were evaluated using Total Lab TL100 software and statistically processed (Figure 3 ). The results obtained from these dot blot analyses on PVDF membranes reflect the fact that treatment by MG132-induced SUMO-2/3 conjugate accumulation in a time dependent manner

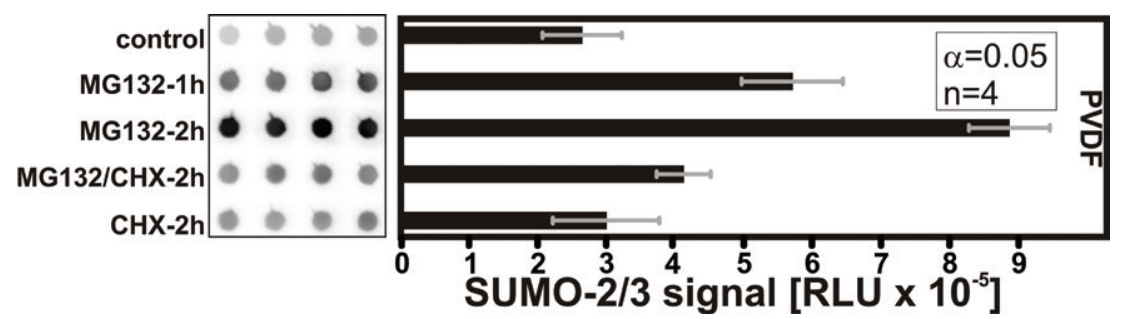

Figure 3. Quantification of changes in global SUMO-2/3 conjugation by dot blot analysis on PVDF membrane. Dot blots were performed on PVDF membranes as described in the Figure 1 legend. HEK $293 \mathrm{~T}$ cells were cultivated on 12 well culture plates: control at $37^{\circ} \mathrm{C}$ (control), translation inhibition by 30 $\mu \mathrm{g} / \mathrm{mL}$ cycloheximide for $2 \mathrm{~h}$ (CHX-2h), proteasomal inhibition by $20 \mu \mathrm{M}$ MG132 for 1 or $2 \mathrm{~h}$ (MG132$1 \mathrm{~h}$ or MG132-2h), and combined translational and proteasomal inhibition (MG132/CHX-2h). For cells subjected to both translational and proteasomal inhibition, the cells were first subjected to inhibition of translation for $20 \mathrm{~min}$ and then exposed to proteasomal inhibitor MG132 for 1 or $2 \mathrm{~h}$ in the presence of the translational inhibitor. Each sample was tested in four replicates and the signal intensity was analyzed statistically and by Total Lab TL100 software.

(Figure 3, MG132-1h and 2h), while treatment with the translational inhibitor cycloheximide strongly decreased the MG132-induced SUMO-2/3 conjugation. This negative effect of cycloheximide on MG132-induced conjugation of SUMO-2/3 in our assay agrees with previous observations $(10,17)$, where SUMO-2/3 deconjugation assays showed that cycloheximide treatment did not influence global cellular SUMO-2/3 levels, but instead affected SUMO-2/3 conju- gation by blocking production of novel SUMOylation targets (17).

As shown above, our approach to evaluate global changes in cellular SUMO-2/3 conjugation is based on increased signal intensity of SUMO-2/3 upon its conjugation. We used this phenomenon to screen for changes in cellular SUMO-2/3 conjugation under different conditions by dot blot analysis on nitrocellulose and PVDF membrane, and showed that the PVDF membrane 
was more suitable for this application. When compared with Western blot, this technique offers the advantages of shorter time requirements, potential to screen large numbers of samples, and ease in evaluating the results using quantification software and statistical analysis. It should be noted that dot blot analysis can be used only to screen for changes in global cellular SUMO-2/3 conjugation; this method is not intended for evaluation of changes in SUMOylation of individual proteins. In those cases, Western blot analysis is still required. Dot blot is particularly useful for comparing SUMO conjugates contained in tissues (in individuals or under different pathological conditions), and screening for activators or inhibitors of SUMO-2/3 conjugation in cell cultures for potential development of drugs modulating SUMO-2/3 conjugation.

\section{Acknowledgments}

This work was funded by the Czech Ministry of Education Research Project grant MSM 6046137305 and by the Financial Support from Specific University Research (MSMT No. 21/2012)

\section{Competing interests}

The authors declare no competing interests.

\section{References}

1. Tatham, M.H., S. Kim, B. Yu, E. Jaffray, J. Song, J. Zheng, M.S. Rodriguez, R.T. Hay, and Y. Chen. 2003. Role of an N-terminal site of Ubc9 in SUMO-1, -2 , and -3 binding and conjugation. Biochemistry 42:9959-9969.

2. Johnson, E.S. 2004. Protein modification by SUMO. Annu. Rev. Biochem. 73:355-382.

3. Kim, J.H. and S.H. Baek. 2009. Emerging roles of desumoylating enzymes. Biochim. Biophys. Acta 1792:155-162.

4. Geiss-Friedlander, R. and F. Melchior. 2007. Concepts in sumoylation: a decade on. Nat. Rev. Mol. Cell Biol. 8:947-956.

5. Saitoh, H. and J. Hinchey. 2000. Functional heterogeneity of small ubiquitin-related protein modifiers SUMO-1 versus SUMO-2/3. J. Biol. Chem. 275:6252-6258.

6. Zhou, W., J.J. Ryan, and H. Zhou. 2004. Global analyses of sumoylated proteins in Saccharomyces cerevisiae. Induction of protein sumoylation by cellular stresses. J. Biol. Chem. 279:32262-32268.

7. Uzunova, K., K. Gottsche, M. Miteva, S.R. Weisshaar, C. Glanemann, M. Schnellhardt, M. Niessen, H. Scheel, et al. 2007. Ubiquitindependent proteolytic control of SUMO conjugates. J. Biol. Chem. 282:34167-34175.

8. Golebiowski, F., I. Matic, M.H. Tatham, C. Cole, Y. Yin, A. Nakamura, J. Cox, G.J. Barton, et al. 2009. System-wide changes to SUMO modifications in response to heat shock. Sci. Signal. 2:ra24.
9. Schimmel, J., K.M. Larsen, I. Matic, M. van Hagen, J. Cox, M. Mann, J.S. Andersen, and A.C. Vertegaal. 2008. The ubiquitin-proteasome system is a key component of the SUMO-2/3 cycle. Mol. Cell. Proteomics 7:2107-2122.

10. Tatham, M.H., I. Matic, M. Mann, and R.T. Hay. 2011. Comparative proteomic analysis identifies a role for SUMO in protein quality control. Sci. Signal. 4:rs4.

11. Lee, Y.J. and J.M. Hallenbeck. 2006. Insights into cytoprotection from ground squirrel hibernation, a natural model of tolerance to profound brain oligaemia. Biochem. Soc. Trans. 34:1295-1298.

12. Lee, Y.J., S. Miyake, H. Wakita, D.C. McMullen, Y. Azuma, S. Auh, and J.M. Hallenbeck. 2007. Protein SUMOylation is massively increased in hibernation torpor and is critical for the cytoprotection provided by ischemic preconditioning and hypothermia in SHSY5Y cells. J. Cereb. Blood Flow Metab. 27:950-962.

13. Yang, W., Q. Ma, G.B. Mackensen, and W. Paschen. 2009. Deep hypothermia markedly activates the small ubiquitin-like modifier conjugation pathway; implications for the fate of cells exposed to transient deep hypothermic cardiopulmonary bypass. J. Cereb. Blood Flow Metab. 29:886-890.

14. Yang, W., H. Sheng, D.S. Warner, and W. Paschen. 2008. Transient global cerebral ischemia induces a massive increase in protein sumoylation. J. Cereb. Blood Flow Metab. 28:269-279.

15. Datwyler, A.L., G. Lättig-Tünnemann, W. Yang, W. Paschen, S.L. Lee, U. Dirnagl, M. Endres, and C. Harms. 2011. SUMO2/3 conjugation is an endogenous neuroprotective mechanism. J. Cereb. Blood Flow Metab. 31:2152-2159.

16. Cimarosti, H., E. Ashikaga, N. Jaafari, L. Dearden, P. Rubin, K.A. Wilkinson, and J.M. Henley. 2012. Enhanced SUMOylation and SENP-1 protein levels following oxygen and glucose deprivation in neurones. J. Cereb. Blood Flow Metab. 32:17-22.

17. Častorálová, M., D. Březinová, M. Švéda, J. Lipov, T. Ruml, and Z. Knejzlík. 2012. SUMO-2/3 conjugates accumulating under heat shock or MG132 treatment result largely from new protein synthesis. Biochim. Biophys. Acta 1823:911-919.

18. Vertegaal, A.C., S.C. Ogg, E. Jaffray, M.S. Rodriguez, R.T. Hay, J.S. Andersen, M. Mann, and A.I. Lamond. 2004. A proteomic study of SUMO-2 target proteins. J. Biol. Chem. 279:33791-33798.

19. Vertegaal, A.C., J.S. Andersen, S.C. Ogg, R.T. Hay, M. Mann, and A.I. Lamond. 2006. Distinct and overlapping sets of SUMO-1 and SUMO-2 target proteins revealed by quantitative proteomics. Mol. Cell. Proteomics 5:2298-2310.

20. Ribet, D., M. Hamon, E. Gouin, M.A. Nahori, F. Impen, H. Neyret-Kahn, K. Gevaert, J. Vandekerckhove, et al. 2010. Listeria monocytogenes impairs SUMOylation for efficient infection. Nature 464:1192-1195.

21. Jones, M.C., L. Fusi, J.H. Higham, H. Abdel-Hafiz, K.B. Horwitz, E.W. Lam, and J.J. Brosens. 2006. Regulation of the SUMO pathway sensitizes differentiating human endometrial stromal cells to progesterone. Proc. Natl. Acad. Sci. USA 103:16272-16277.
Received 30 April 2012; accepted 13 August 2012.

Address correspondence to Zdeněk Knejzlík, Department of Biochemistry and Microbiology, Institute of Chemical Technology, Prague, Technická 5, 16628 Prague, Czech Republic. Email:Zdenek.Knejzlik@vscht.cz

To purchase reprints of this article, contact: biotechniques@fosterprinting.com 